\title{
Diagnostic accuracy of double inversion recovery in delineation of multiple sclerosis lesions and its clinical correlation with expanded disability scoring system
}

\author{
Weaam Hamed ${ }^{1 *}$, Wessam Fathi ${ }^{2}$, Walaa Mahmoud ${ }^{1}$ and Galal Elhawary ${ }^{1}$ (D)
}

\begin{abstract}
Background: The aim of our work was to assess the diagnostic accuracy of a double inversion recovery (DIR) sequence in the detection of brain and spinal cord MS lesions. In addition, we aimed to evaluate the reliability of the correlation between GM affection and high Expanded Disability Status Scale (EDSS) to enhance the role of DIR as a practical test for clinical disability. Ninety MS patients were prospectively included in this study. Imaging was performed using Philips Intera 1.5 Tesla device. T2W-TSE, FLAIR, and DIR sequences were performed and compared to each other. Each patient was clinically assessed at the time of the MRI examination with EDSS.

Results: DIR showed significantly higher total lesion load in comparison to T2 and FLAIR with increased sensitivity for cortical lesion detection by DIR. There was a positive association between patients with cortical lesions and male gender, clinical disability, cognitive changes, and higher EDSS score.

Conclusion: The increased rate of cortical lesion detection by DIR does not affect its accuracy in white matter lesions count. Moreover, DIR provided a better morphological characterization and delineation of white matter lesion with good differentiation between juxtacortical and mixed white matter-gray matter lesions; thus, we recommend adding DIR sequence in routine MR protocols for MS patients.
\end{abstract}

Keywords: Double inversion recovery (DIR), Expanded disability status scale (EDSS), Multiple sclerosis (MS), Normalappearing white matter (NAWM), Normal-appearing gray matter (NAGM)

\section{Background}

Multiple sclerosis (MS) is the most frequent chronic demyelinating disorder of the central nervous system [1]. Not only it affects mainly the white matter (WM) but also it affects parts of the gray matter (GM) [2]. Recent studies have revealed correlations between the GM lesions and the degree of physical and cognitive dysfunction [3]. Therefore, it was important to assess GM lesion load as well as cognitive disability and correlate between them.

Over $50 \%$ of MS patients suffer from neuropsychological impairment such as deficits in processing speed

\footnotetext{
* Correspondence: weaam.hamed@gmail.com

'Department of Diagnostic and Interventional Radiology, Faculty of

Medicine, Mansoura University, Mansoura 35516, Egypt

Full list of author information is available at the end of the article
}

and memory. A cognitive impairment significantly impacts the lives of patients with MS and their families. Identifying patients at risk for cognitive impairment on the basis of MRI would enhance the quality of care [4].

The assessment of GM has substantially been improved by advanced magnetic resonance imaging (MRI) techniques like double inversion recovery (DIR). This sequence produces two different inversion pulses, which attenuates the CSF together with the whole white matter, thus providing a remarkable delineation between gray and white matter [5]. DIR improves the visibility of gray matter particularly cortical MS lesions in comparison to standard MRI techniques [3]. In addition to its important role of DIR in MS, it also used in the detection of focal cortical dysplasia (FCD) in children and young adults with epilepsy [6]. 
From the clinical point, the degree of neurologic impairment and the level of disability in MS patients are assessed by the Expanded Disability Status Scale (EDSS) which is the most widely used scale nowadays [7]. EDSS is a 20-step scale of disease severity ranging from 0 (normal) to 10 (death due to MS). This scale includes two parts [8]:

A. (From 0 to 3.5) taking into account functional parameter.

B. (From 4 to 10) estimating degrees of mobility in patients.

\section{Aim of the work}

The aim of our work was to assess the diagnostic value of a DIR sequence in the detection of MS lesions by comparing DIR with FLAIR and T2-weighted pulse sequences in different anatomical regions in CNS. In addition, we aimed to evaluate the reliability of the correlation between GM affection and high EDSS score to enhance the role of DIR as a practical test for clinical disability.

\section{Methods}

Ninety patients were prospectively included in this study (ranged from 15 to 51 years with the mean age was $33.24 \pm 8.93)$. They were already diagnosed to have multiple sclerosis according to the 2017 revised McDonald criteria [3]. The majority of our cases were relapsingremitting multiple sclerosis (RRMS) with a percentage of 91.1\%, 5.6\% were secondary-progressive multiple sclerosis (SPMS), and only 3.3\% were primary-progressive multiple sclerosis (PPMS). All the patients were referred from the neurology department to the MRI unit of the radiology department at Mansoura University hospitals, in the period from January 2017 till March 2018. Each patient was clinically assessed at the time of the MRI examination with EDSS. This study was approved by our institution's ethics committee, and all patients gave their consent before inclusion in the study.

\section{MR imaging}

Imaging was performed at $1.5 \mathrm{~T}$ MRI (Philips Intera) using a standard head coil for the brain and standard head and neck coil of the cervical spine, and patients were in a supine position. T2W-TSE, FLAIR, and DIR sequences were performed. No post-contrast acquisitions were done in our study as we were concerned with the true anatomical delineation of MS plaques not to detect disease activity. The three sequences were compared in the axial planes as regard brain lesions with identical anatomic position, using parameters including field of view (FOV), matrix, voxel size, and slice thickness which were summarized in Table 1 . DIR and T2W-TSE were
Table 1 Brain and cervical spine MRI sequences parameters

\begin{tabular}{llll}
\hline Brain sequences parameters & & & \\
Parameter & T2W-TSE & FIAIR & DIR \\
Slice orientation & Axial & Axial & Axial \\
Flied of view (mm) & 230 & 230 & 230 \\
Matrix & 256 & 256 & 256 \\
Slice thickness (mm) & 5 & 5 & 5 \\
Voxel size & 0.9 & 0.9 & 0.9 \\
Repetition time (ms) & 4500 & 10,000 & 9600 \\
Echo time (ms) & 100 & 140 & 25 \\
Inversion time (ms) & - & 2800 & $3400 / 325$ \\
Spine sequences parameters & & \\
Parameter & T2W-TSE & DIR & \\
Slice orientation & Sagittal & Sagittal & \\
Matrix & $256 \times 256$ & $208 \times 208$ & \\
Voxel size & $1.2 \times 1.2 \times 1.3$ & $1 \times 1.2 \times 2$ & \\
Repetition time (ms) & 3000 & 5500 & \\
Echo time (ms) & 120 & 25 & \\
Inversion time (ms) & - & $2550 / 450$ & \\
\hline
\end{tabular}

compared in sagittal planes as regard sagittal with the following parameters which were summarized also in Table 1.

From our practice, the sagittal plane is preferred to display MS lesions in calloso-septal interface and corpus callosum areas. But MS plaques in the areas of cortical and juxtacortical are demonstrated better in axial orientation; thus, we prefer to study MS plaques in the three sequences in the axial view.

\section{MR imaging interpretation}

The images were interpreted by conjoint analysis done by two radiologists with 4 and 10 years of experience; they were blinded to EDSS results.

MS lesions were identified as hyper-intense spots observed in T2, FLAIR, and DIR images with a size of $\geq 2$ $\mathrm{mm}$. In contrast to these hyper-intense spots, there was some high signal intensity in a striped shape known as flow artifacts in extra cortical regions rising from the sinuses or major vessels which are considered artifacts and not lesions.

The detected lesions were classified according to their anatomical locations into six regions: cortical, juxtacortical, deep white matter, mixed white matter-gray matter, periventricular white matter, and infra-tentorial regions. Then, the lesion numbers were calculated in these regions in each sequence.

The signal intensity of the lesion was determined by locating the region-of-interest (ROI) in a mean size of $3 \times 3 \mathrm{~mm}$ on the lesions as well as the signal intensity of normal-appearing white matter (NAWM), normal- 
appearing gray matter (NAGM), and CSF. Then, the signal ratios were calculated from the mean values of the signal intensity among lesions/NAGM, lesions/NAWM, and lesions/CSF separately in each technique and all anatomical regions. The signal ratios were assessed according to (SI1 - SI2)/(SI1 + SI2), in which SI1 represents the signal intensity of the lesions and SI2 represents the signal intensity of the NAWM, NAGM, or CSF.

As regard spinal cord evaluation, the lesions were classified as "present" or "absent" and not as a lesion count. The signal ratio was not applied on a spinal cord lesion as it had no significant difference between two sequences.

\section{Statistical analysis}

Statistical analysis data were analyzed with SPSS version 21. The normality of data was first tested with a onesample Kolmogorov-Smirnov test; qualitative data were described using number and percent. Association between categorical variables was tested using chi-square test. Significant variables entered into logistic regression model using the entered statistical technique to predict the most significant determinants and to control for possible interactions and confounding effects.

For all the abovementioned statistical tests done, the threshold of the significance is fixed at 5\% level. The results were considered significant when the probability of error is less than $5 \%$ and highly significant when the probability of error is less than $0.1 \%$.

\section{Results}

The percentage of cases with gray matter affection was $45.6 \%$. The lesions were classified anatomically into supratentorial and infratentorial in location. The supratentorial lesions were further categorized into peri-ventricular WM, deep WM, juxtacortical, mixed gray-white matter, and cortical lesions.

In Table 2, we found that DIR sequence was significantly superior to $\mathrm{T} 2$ as regards the number of lesions detected in four anatomical regions (PVWM, mixed W-GM, cortical, and infra-tentorial) with relative gain 31\%, 47\%, $98 \%$, and $39 \%$, respectively. However, there is no statistically significant difference between the two sequences as regard DWM and JC lesion detection. Regarding the total lesion load, we found that DIR was significantly superior to T2 $(P=0.003$ with a relative gain of $18 \%)$.

In comparison to FLAIR, we found that the DIR sequence was significantly superior to FLAIR as regards the number of lesions detected in three anatomical regions (mixed W-GM, cortical, and infra-tentorial) with relative gain $24 \%, 80 \%$, and $63 \%$, respectively. However, there is no statistically significant difference between the two sequences as regard PVWM, DWM, and JC lesion detection. Regarding the total lesion load, we found that DIR was significantly superior to FLAIR ( $P=0.039$ with a relative gain of 7\%) (Figs. 1 and 2).

Regarding the signal ratio between the lesion to the background, Table 3 showed that the signal between lesions against NAWM was significantly higher in DIR images compared to T2 and FLAIR in different anatomical locations (IT, PVWM, DWM, and JC) $(P<0.001)$, The contrast between the lesions and CSF was significantly higher in DIR compared to T2 $(P<0.001)$, but it was without statistical significance when compared to FLAIR $(P=0.071)$.DIR showed slightly higher contrast between the lesions against NAGM, when compared to the

Table 2 Analysis of the lesion load measurement and relative comparisons of DIR versus T2 and DIR versus FLAIR sequences

\begin{tabular}{|c|c|c|c|c|c|c|c|}
\hline \multirow[t]{2}{*}{ Region } & \multirow[t]{2}{*}{ DIR } & \multirow[t]{2}{*}{$\mathrm{T} 2$} & \multirow[t]{2}{*}{ FLAIR } & \multicolumn{4}{|c|}{ Relative ratio (\%) } \\
\hline & & & & $\mathrm{DIR} / \mathrm{T} 2$ (\%) & $P$ value & DIR/FLAIR (\%) & $P$ value \\
\hline PWWM & $\begin{array}{l}12.6 \pm 3.9 \\
13(0-41)\end{array}$ & $\begin{array}{l}9.6 \pm 3.1 \\
7.5(0-35)\end{array}$ & $\begin{array}{l}12.1 \pm 4.1 \\
8.5(0-37)\end{array}$ & $31 \%$ & $0.033^{*}$ & $4 \%$ & 0.927 \\
\hline DWM & $\begin{array}{l}10.2 \pm 3.8 \\
8(1.0-34)\end{array}$ & $\begin{array}{l}10.3 \pm 3.5 \\
10.0(0-38)\end{array}$ & $\begin{array}{l}10.4 \pm 3.3 \\
9.0(0-34)\end{array}$ & $-1 \%$ & 0.834 & $-3 \%$ & 0.619 \\
\hline Mixed W-GM & $\begin{array}{l}9.1 \pm 3 \\
8(0-25)\end{array}$ & $\begin{array}{l}6.2 \pm 2.7 \\
5.00(0-21)\end{array}$ & $\begin{array}{l}7.3 \pm 2.1 \\
6.0(1.0-23)\end{array}$ & $47 \%$ & $<0.001^{* *}$ & $24 \%$ & $0.009^{*}$ \\
\hline$J C$ & $\begin{array}{l}9.1 \pm 2.6 \\
6(0-22)\end{array}$ & $\begin{array}{l}9.6 \pm 2.1 \\
5.00(0-25)\end{array}$ & $\begin{array}{l}10 \pm 2.2 \\
6.0(0-21)\end{array}$ & $-2 \%$ & 0.696 & $-5 \%$ & 0.622 \\
\hline Cortical & $\begin{array}{l}1.5 \pm 0.8 \\
1(0-9)\end{array}$ & $\begin{array}{l}0.6 \pm 0.2 \\
0.0(0-7)\end{array}$ & $\begin{array}{l}0.8 \pm 0.2 \\
0(0-7.0)\end{array}$ & $98 \%$ & $0.003^{*}$ & $80 \%$ & $0.004^{*}$ \\
\hline IT & $\begin{array}{l}1.9 \pm 0.5 \\
2.0(0-15)\end{array}$ & $\begin{array}{l}1.5 \pm 0.3 \\
1.0(0-9)\end{array}$ & $\begin{array}{l}1.2 \pm 0.3 \\
1.0(0-8.0)\end{array}$ & $39 \%$ & $0.023^{*}$ & $63 \%$ & $0.002^{*}$ \\
\hline Total & $\begin{array}{l}41.9 \pm 13.4 \\
52(6-121)\end{array}$ & $\begin{array}{l}35 \pm 9.6 \\
31.5(3-107)\end{array}$ & $\begin{array}{l}38.7 \pm 12.4 \\
33.5(4-114)\end{array}$ & $18 \%$ & $0.003^{*}$ & $7 \%$ & $0.039^{*}$ \\
\hline
\end{tabular}

Data were expressed as mean \pm SD and median (range)

$P$ probability

*Statistically significant $p<0.05$

**Highly significant $p<0.001$ 

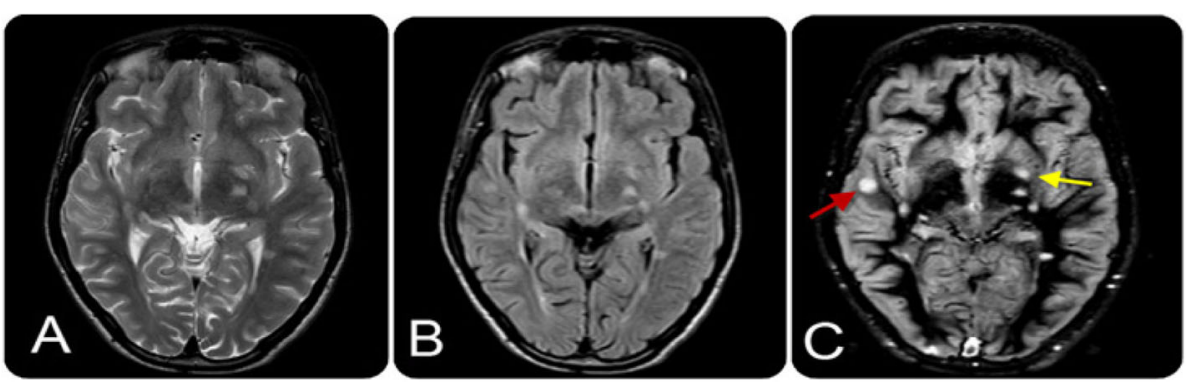

Fig. 1 a T2, b FLAIR, c DIR axial brain cuts in male 36 years old with history of RRMS since 7 years, presented by weakness of both $L L$, migraine and vertigo, and $\mathrm{EDSS}=6$, showing a more sharp delineated hyper-intense cortical lesion (red arrow) seen on DIR (c) compared to its faint appearance on T2WI (a) and FLAIR (b). Another hyper-intense lesion seen affecting left globus pallidus of lentiform nucleus (deep gray matter affection) (yellow arrow) with better anatomical characterization and delineation on DIR (c) compared to other sequences. A better contrast and delineation of the other detected lesions on DIR (c) compared to other images

FLAIR and T2; but still, these measurements were below statistical significance $(P=0.169$ and 0.221 , respectively) (Fig. 1).

Regression analysis was conducted for the prediction of cortical lesions within MS patients using age, gender, and duration as covariates. Males and longer duration were considered as independent risk factors for cortical lesions within MS patients in uni- and multivariable analyses (Table 4).

Regarding the association between the clinical outcome of MS patients and cortical affection, we found that $92.7 \%$ of patients with cortical affection exhibited a higher EDSS score $(>4)$ and associated with more disability (Table 5 and Fig. 3). There was a statistically significant association between cortical affection and higher EDSS with $P<0.001$ and sensitivity $92.5 \%$ and specificity 94\% (Fig. 4).

With regard to spinal lesions, they were observed in 31 out of 90 MS patients with a percentage $34.4 \%$ (Fig. 5).

\section{Discussion}

MS has classically been described as a WM disorder [9]; however, over the few past years, an increased attention has been pointed toward the involvement of GM in the pathophysiology of MS [10]. The acknowledgment of GM involvement in MS has led to the incorporation of cortical/juxtacortical lesions in the recent 2017 revised McDonald's diagnostic criteria for MS [3]. Since abnormalities in cortical gray matter have been correlated with both physical and neuropsychological deficits in MS patients, it is essential to create a better assessment and a more accurate estimation of gray matter lesion load in vivo [10].

The majority of our patients (91.1\%) were categorized according to their clinical course as RRMS, while only $5.6 \%$ were categorized as SPMS and 3.3\% were categorized as PPMS. This is in consensus with the fact that RRMS is the most common disease course.

DIR detected significantly more cortical lesions in our patients when compared to T2 $(P$ value 0.003 , relative
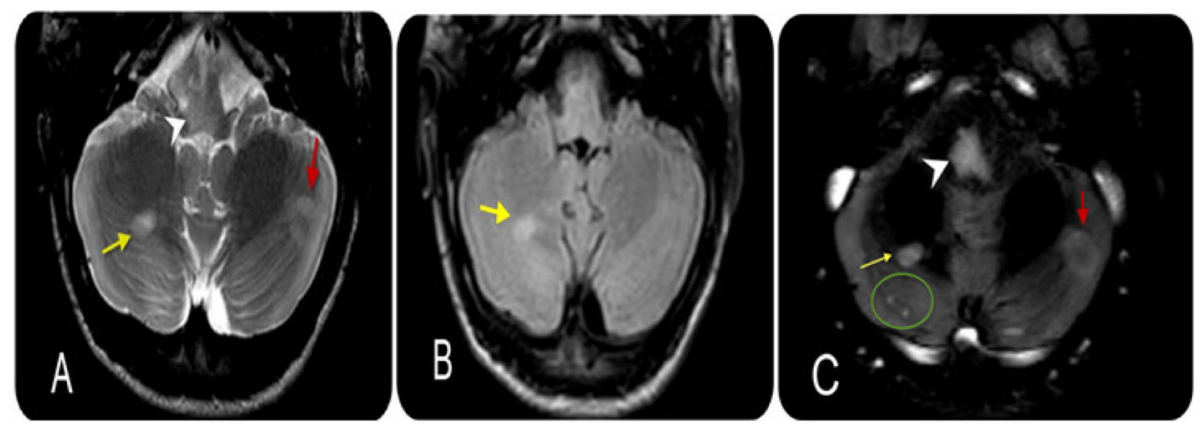

Fig. 2 a T2, b FLAIR, c DIR axial brain cuts in a male patient 21 years old with history of RRMS since 2 years, presented by vertigo, diminution of vision, dysphagia, and EDSS $=4.5$, shows large cortical lesion (red arrow) is seen encompassing several folia in the left cerebellar hemisphere that is identified by both T2WI (a) and DIR (c) and not seen on FLAIR image (b), with two small spot-like cortical lesions (surrounded by circle) observed in the right hemisphere, detected only by DIR (c). Another mixed white-gray matter lesion (yellow arrow) seen in the right cerebellar hemisphere which is recognized by all sequences. The arrowhead points out a lesion in the medulla oblongata which is better delineated on DIR (c), faintly detected on T2WI (a) and not visualized on FLAIR (b) 
Table 3 Comparison of signal ratios between DIR versus FLAIR and DIR versus T2 in all studied cases. Data were expressed as median (range)

\begin{tabular}{llllll}
\hline Signal ratio & DIR & T2-WI & $P$ value & FLAIR & $P$ value \\
\hline Lesion/NAWM (IT) & $0.65(0.52 / 0.82)$ & $0.16(0.07 / 0.25)$ & $<0.001^{* *}$ & $0.130 .10 / 0.34)$ & $<.18(0.12 / 0.28)$ \\
Lesion/NAWM (PWWM) & $0.845(0.74 / 0.98)$ & $0.21(0.06 / 0.36)$ & $<0.001^{* *}$ & $<0.01^{* *}$ \\
Lesion/NAWM (DWM) & $0.92(0.89 / 0.97)$ & $0.06(0.04 / 0.08)$ & $<0.001^{* *}$ & $0.24(0.20 / 0.30)$ & $<0.001^{* *}$ \\
Lesion/NAWM (JC) & $0.78(0.60 / 0.98)$ & $0.14(0.09 / 0.19)$ & $<0.001^{* *}$ & $0.16(0.11 / 0.21)$ & $<0.001^{* *}$ \\
Lesion/CSF & $0.67(0.54 / 0.84)$ & $0.003(-0.02 / 0.02)$ & $<0.001^{* *}$ & $0.60(0.43 / 0.77)$ & $0.001^{* *}$ \\
Lesion/NAGM & $0.08(0.05 / 0.22)$ & $0.07(0.05 / 0.22)$ & 0.221 & $0.09(-0.09 / 0.23)$ & 0.169 \\
\hline
\end{tabular}

**Highly significant value

gain about $98 \%)$ and FLAIR ( $P$ value 0.004 relative gain about 80 ). This was equivalent to studies done by Geurts et al. [10] and Elnekeidy et al. [11].

Besides the increased sensitivity to cortical lesions, we found the second major advantage of DIR imaging is its apparent potential for better differenation between mixed white matter-gray matter lesions and juxtacortical lesions. In our study, some of the lesions which were scored as juxtacortical on T2 and FLAIR images often turned out to be mixed white matter-gray matter lesions on DIR images. This is supported by the observation of a reduced number of juxtacortical lesions scored on DIR images compared with T2 $(P=0.696$ relative loss about $2 \%)$ and FLAIR $(P=0.622$, relative loss about $5 \%)$.

On the other hand, there was a statistically significant increase in mixed white matter-gray matter lesions detection on DIR imaging at the expense of T2 $(P<0.001$ relative gain about $47 \%)$ and FLAIR $(P=0.009$, relative gain about $24 \%$ (.The acceptable explanation for these different detection rates in the juxtacortical lesions and mixed white matter-gray matter lesions is the sharp delineation between gray and white matter on DIR, which allowed strict differentiation between purely juxtacortical lesions and lesions already touching the cortical area (Fig. 2).

As regard juxtacortical lesions, our results were consistent with the findings of the study done by Wattjes et al. [5]. However, our results were in contrary with the results done by Geurts et al. [10] which detected the highest number of lesions with T2. Regarding mixed white matter-gray matter lesions, our study was consistent with the reported results of Vural et al. [1].

Another benefit of DIR in our study was its ability to detect periventricular white matter (PVWM) lesions. DIR detected significantly more lesions in comparison with T2 $(P=0.033$, relative gain about 31$)$. Not surprisingly, DIR detected lesions with a slightly higher difference in comparison to FLAIR; this difference was below the statistical importance $(P=0.927$, relative gain about $4 \%)$. The converged results between DIR and FLAIR return to CSF signal nulling in both sequences. However, we must point that DIR added a better morphological characterization and delineation of lesions which become more countable compared with confluent configuration in FLAIR and T2. Our results were equivalent to the reported results from Abidi et al. [2] and Wattjes et al. [5] but inconsistent with the reported results from Elnekeidy et al. [11] who found that no significant difference in lesion load between DIR and $\mathrm{T} 2$.

In the present study, we concluded almost similar detection rates as regard deep white matter (DWM) lesions by all sequences, with no statistically significant difference between them. Our results correlated to the results reported by Abidi et al. [2]; however, our results were in

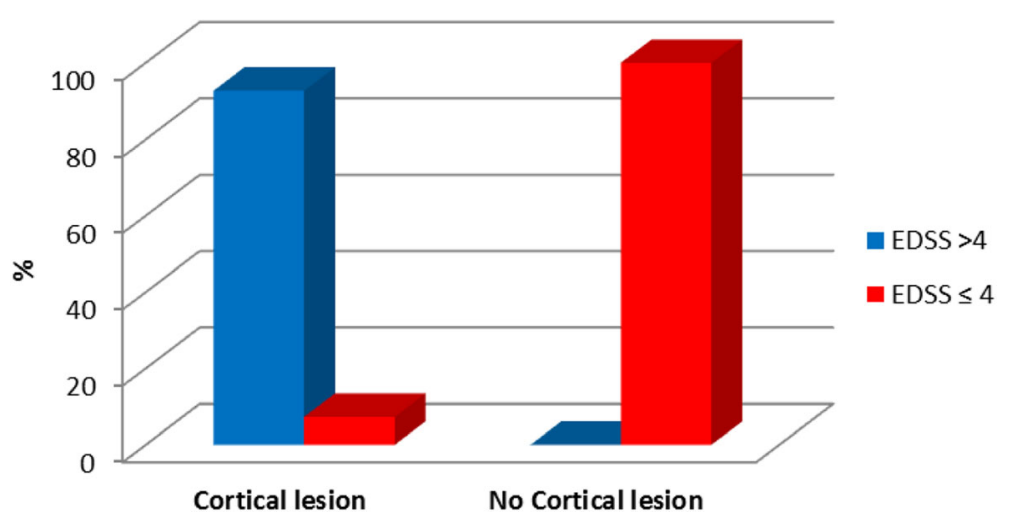

Fig. 3 Relationship between cortical affection and clinical outcome (EDSS) of the patients 
Table 4 Regression analysis for prediction of cortical lesions within MS patients

\begin{tabular}{|c|c|c|c|c|c|c|c|c|}
\hline \multirow[b]{3}{*}{ Age } & \multicolumn{4}{|c|}{ Univariate } & \multicolumn{4}{|c|}{ Multivariate } \\
\hline & \multirow{2}{*}{$\frac{P}{0.760}$} & \multirow{2}{*}{$\frac{\mathrm{OR}}{1.003}$} & \multicolumn{2}{|l|}{$95 \% \mathrm{Cl}$} & \multirow{2}{*}{$\begin{array}{l}P \\
-\end{array}$} & \multirow{2}{*}{$\frac{\mathrm{OR}}{-}$} & \multicolumn{2}{|l|}{$95 \% \mathrm{Cl}$} \\
\hline & & & .958 & 1.051 & & & - & - \\
\hline Males & $<0.001$ & 4.899 & 2.651 & 9.053 & $<0.001$ & 4.096 & 2.149 & 7.807 \\
\hline Duration & 0.003 & 1.146 & 1.053 & 1.249 & 0.049 & 1.486 & 1.191 & 1.792 \\
\hline
\end{tabular}

$O R$ odds ratio, $\mathrm{Cl}$ confidence interval, logistic regression analysis was used

contrary with the results done by Elnekeidy et al. [11] which detected a higher number of lesions by DIR in comparison to T2 and FLAIR.

Another important advantage of DIR in our study was its ability to detect infra-tentorial lesions. DIR identified significantly more lesions in comparison to FLAIR $(P=$ 0.002 , relative gain about $63 \%$ ). But, it was worthy of attention that DIR detected higher lesions even when compared with the T2, which is considered the "gold standard" in the infra-tentorial region $(P=0.023$, relative gain about 39\%).This was consistent with the results done by Abidi et al. [2] and by Elnekeidy et al. [11] but in contrary with results done by Moraal et al. [12] who found a similar number of lesions in the infra-tentorial region with DIR, FLAIR, and T2 images.

On the light of previously discussed results of diagnostic accuracy of DIR in different anatomical locations in comparison with T2 and FLAIR, we concluded that the increased rate of cortical lesion detection by DIR does not affect its accuracy in white matter lesions count. Moreover, DIR provided a better morphological characterization and delineation of white matter lesion with good differentiation between juxtacortical and mixed white matter-gray matter lesions. This means that DIR can be useful for clinical purposes as a supplement to or even as a replacement for standard T2 and FLAIR.

In our study, lesion-to-background signal ratio was calculated objectively in order to avoid creating subjective data by visual evaluation of signal ratio, which may be affected by windows and levels, magnification, and monitor brightness. We demonstrated a significantly higher signal ratio of lesions to NAWM on the DIR in comparison with T2W and FLAIR in all anatomical regions (infra-tentorial, periventricular, juxtacortical, and DWM) with $(P<0.001)$ which allowed better delineation of lesions by DIR. The higher contrast ratio detected by DIR can be explained by its complete suppression of white matter signal. Furthermore, the contrast ratio of lesions to CSF was significantly higher in DIR compared to T2 $(P<0.001)$. However, there was no statistically significant difference between the DIR and FLAIR regarding the contrast of lesion compared to CSF $(P=0.071)$ which was explained by CSF nulling by both sequences. It is important to mention that CSF suppression on DIR was less homogenous in some of our cases, but it was suitable for lesion detection even in the worst conditions.

On the other hand, there was no statistically significant difference between DIR and both FLAIR and T2 as regards the signal ratio of lesion to NAGM which explained that the three sequences have the same gray matter signal intensity. But, it was worthy of attention that the DIR allowed better delineation of gray matter.

The diagnosis of MS is based on the 2017 revised McDonald's criteria which depend on the demonstration of lesion dissemination in both space and time. On MRI, the detection of at least one lesion on the spinal cord in addition to at least one cerebral lesion can fulfill the dissemination in space criteria [3]. Therefore, it was important to assess if there is an added value of DIR in spinal cord lesion detection.

In our work, Spinal lesions were observed in 31 out of 90 MS patients (34.4\%). The importance of DIR in the spinal cord lesion detection is highlighted by three cases, in which a definite diagnosis of MS was highly suspected clinically, but not confirmed by radiology yet. One of them had a prominent lesion in the cervical cord by both T2 and DIR; however, a detection of a second lesion that is only visible in DIR largely excluded a neoplastic process and favored the diagnosis of an inflammatory disease.

In the other two cases, spinal cord lesion was only revealed by DIR that helped us to complete the criteria of dissemination in space, changing the diagnosis from "possible" to "definite" MS. In the rest of the cases, there was no difference between DIR and T2 as regard the number of detected lesions. However, the lesion-tobackground contrast was higher in DIR that provided better lesion visualization.

Our previously discussed results were quite promising as we found that DIR provided a more exact delineation

Table 5 Relationship between cortical affection and clinical outcome (EDSS) of the patients

\begin{tabular}{|c|c|c|c|c|c|c|}
\hline \multirow[t]{2}{*}{ EDSS } & \multicolumn{2}{|c|}{ Cortical lesion $(n=41)$} & \multicolumn{2}{|c|}{ No cortical lesion $(n=49)$} & \multirow{2}{*}{$\begin{array}{l}\text { Test of } \\
\text { significance }\end{array}$} & \multirow[t]{2}{*}{$P$ value } \\
\hline & $\mathrm{No}$ & $\%$ & No & $\%$ & & \\
\hline$>4$ & 38 & 92.7 & 0 & 0.0 & $x^{2}=15.71$ & $<0.001^{* *}$ \\
\hline$\leq 4$ & 3 & 7.3 & 49 & 100.0 & & \\
\hline Median (range) & \multicolumn{2}{|c|}{$5.50(2.00-8.50)$} & \multicolumn{2}{|c|}{$2.50(1.00-3.50)$} & $Z=4.33$ & $<0.001^{* *}$ \\
\hline
\end{tabular}

**Highly significant value 


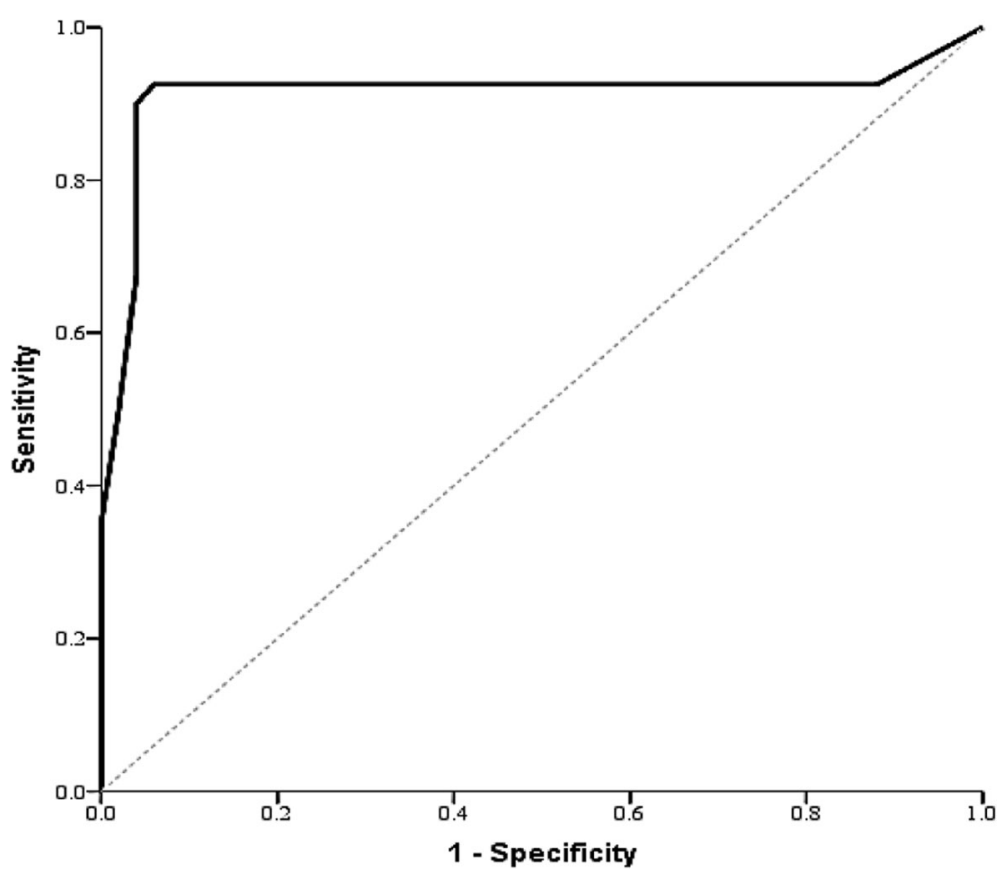

Fig. 4 ROC curve of EDSS score in patients with cortical lesions

of spinal cord lesions and slightly improved lesiondetection rates.

Since cortical lesions are associated with more diffuse and severe pathologic process resulting in poor clinical prognosis, it was an important aspect to evaluate associated factors for developing cortical lesions. Cortical lesions were detected in $45.6 \%$ of patients, 26 were males and 15 of them

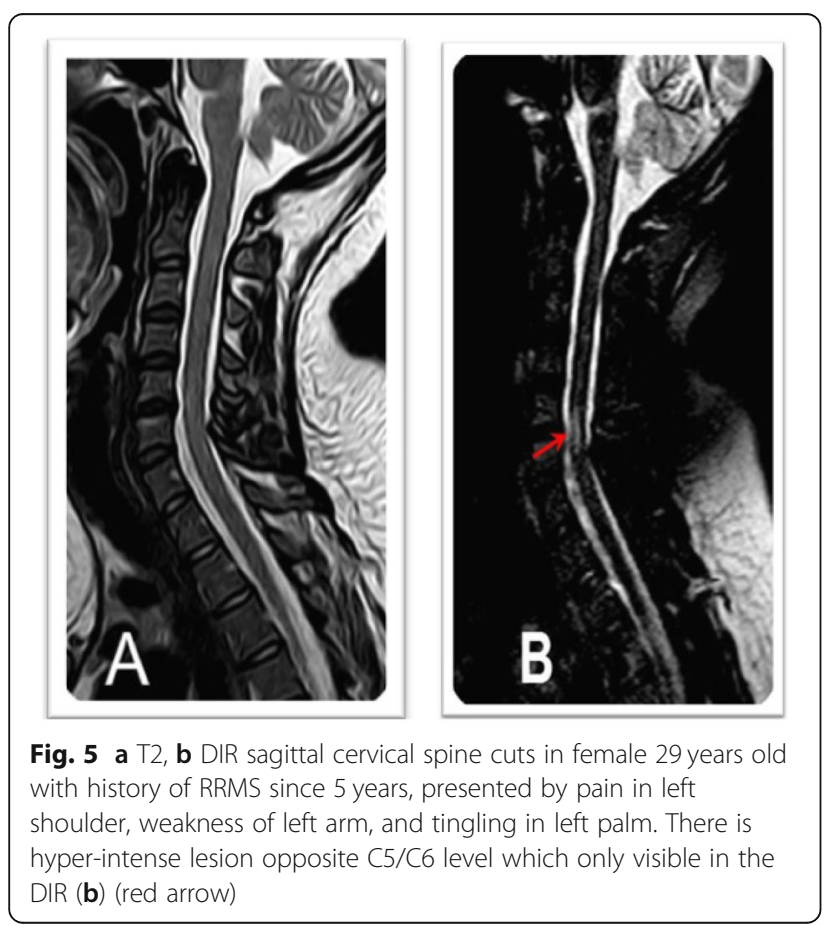

were females. Therefore, males were significantly more at risk to have cortical lesions $(P<0.001)$. The predominance of cortical lesions in males deserved attention as despite higher prevalence of MS in females, the poor prognosis was associated with male gender. Our results correlated with the study done by Bergamaschi [13] which reported that male sex is a negative prognostic factor for MS, as well as the study done by Calabrese et al. [14] which confirmed the same results. However, our results were in contrary to the study done by Tremlett et al. [15] which reported that the male sex was not associated with worse disease outcome.

In our study, we found that cortical lesions were significantly more frequent and numerous at later MS stages (disease duration $>10$ years) with $(P=0.003)$. This was in agreement with the results done by Elnekeidy et al. [11] and Calabrese et al. [14]. But, we cannot ignore that cortical lesions were also observed in few cases in earlier stages of the disease (disease duration $<5$ years). The observation of cortical lesions early proved that the GM affection in MS does not always occur as a secondary change to WM affection, but may occur as a result of independent pathology of the GM.

From the clinical aspect, we observed that patients with cortical lesions presented clinical disability, cognitive changes, and higher EDSS score. There was a significant correlation between cortical lesions and EDSS $(P<$ 0.001). This was consistent with the finding of the study done by Calabrese et al. [14] and Vural et al. [1]. The positive association of clinical outcome and cortical lesion load which was detected by DIR is considered a 
great advantage of the DIR sequence. It helps us to overcome the "clinico-radiological paradox" in MS which refers to the weak correlation of conventional MRI lesion load with clinical disability due inability of conventional MRI techniques to demonstrate all of the histopathological changes present in MS.

The main limitation in our study was DIR-related artifacts which were observed at posterior fossa, choroid plexus, and periventricular WM that may be from CSF pulsation or from sinuses and bigger vessels, and in addition to that, there were some bilateral high-signal ribbon-like artifacts which were observed in extra-cortical regions and their appearance changed in continual sections. To overcome that, we observed multiple slices and other MRI sequences such as T2 and FLAIR to help us to differentiate lesions from artifacts.

Another limitation in our study is that we were limited to perform 2D-DIR in spite of the advantages of 3D-DIR because of the high scan time of 3D-DIR. Therefore, we compared 2D-DIR in optimal parameters with 2DFLAIR and 2D-T2W-TSE.

\section{Conclusion}

DIR can be useful for clinical purposes as a supplement to or even as a replacement for standard T2 and FLAIR as well as in explanation of physical and cognitive dysfunction.

\section{Abbreviations}

DIR: Double inversion recovery; DWM: Deep white matter; EDSS: Expanded disability status scale; FCD: Focal cortical dysplasia; FOV: Field of view; GM: Gray matter; IT: Infra-tentorial; JC: Juxtacortical; MRI: Magnetic resonance imaging; MS: Multiple sclerosis; NAGM: Normal-appearing gray matter; NAWM: Normal-appearing white matter; PPMS: Primary-progressive multiple sclerosis; PWWM: Peri-ventricular white matter; ROI: Region-of-interest; RRMS: Relapsing-remitting multiple sclerosis; SPMS: Secondary-progressive multiple sclerosis; WM: White matter

\section{Acknowledgements}

Not applicable

\section{Authors' contributions}

WH was responsible on imaging the cases and arranged radiological data on the sheets. WM revised the radiological data and performed the statistical analysis. WF carried out the neurological assessment to the cases. GE drafted the manuscript. All authors read and approved the final manuscript.

\section{Funding}

No funding was obtained for this study.

\section{Availability of data and materials}

The datasets used and analyzed during the current study are available from the corresponding author on reasonable request.

\section{Ethics approval and consent to participate}

This study was approved by the research ethics committee of the Faculty of Medicine at Mansoura University in Egypt on 8 January 2017, reference number of approval: MS/16.12.84, and all patients included in this study gave written informed consent to participate in this research.

\section{Consent for publication}

All patients included in this research gave written informed consent to publish the data contained within this study. If the patient was less than 16 years old or unconscious when consent for publication was requested, written informed consent for the publication of this data was given by their parent or legal guardian.

\section{Competing interests}

The authors declare that they have no competing interests.

\section{Author details}

'Department of Diagnostic and Interventional Radiology, Faculty of Medicine, Mansoura University, Mansoura 35516, Egypt. ${ }^{2}$ Department of Neurology, Faculty of Medicine, Mansoura University, Mansoura, Egypt.

Received: 30 June 2019 Accepted: 24 September 2019

Published online: 30 December 2019

\section{References}

1. Vural G et al (2013) Comparison of double inversion recovery and conventional magnetic resonance brain imaging in patients with multiple sclerosis and relations with disease disability. Neuroradiol J 26(2):133-142

2. Abidi Z et al (2017) Assessment of the diagnostic accuracy of double inversion recovery sequence compared with FLAIR and T2W_TSE in detection of cerebral multiple sclerosis lesions. Electron Physician 9(4):4162-4170

3. Thompson AJ et al (2017) Diagnosis of multiple sclerosis: 2017 revisions of the McDonald criteria. Lancet Neurol 17(2):162-173

4. Almolla RM et al (2016) Correlation of apparent diffusion coefficient to cognitive impairment in relapsing remittent multiple sclerosis (plaque, periplaque and normal appearing white matter). Egypt J Radiol Nucl Med 47(3): 1009-1018

5. Wattjes MP et al (2007) Double inversion recovery brain imaging at $3 \mathrm{~T}$ : diagnostic value in the detection of multiple sclerosis lesions. Am J Neuroradiol 28(1):54-59

6. Soares BP et al (2016) Utility of double inversion recovery MRI in paediatric epilepsy. Br J Radiol 89(1057):20150325

7. Meyer-Moock S et al (2014) Systematic literature review and validity evaluation of the Expanded Disability Status Scale (EDSS) and the Multiple Sclerosis Functional Composite (MSFC) in patients with multiple sclerosis. BMC Neurol 14(1):58

8. Guzel I et al (2016) Is there an association between the Expanded Disability Status Scale and inflammatory markers in multiple sclerosis? I Chin Med Assoc 79(2):54-57

9. Frischer JM et al (2015) Clinical and pathological insights into the dynamic nature of the white matter multiple sclerosis plaque. Ann Neurol 78(5):710-721

10. Geurts JJG et al (2005) Intracortical lesions in multiple sclerosis: improved detection with 3D double inversion-recovery MR imaging. Radiology 236(1):254-260

11. Elnekeidy AM et al (2014) Added value of double inversion recovery magnetic resonance sequence in detection of cortical and white matter brain lesions in multiple sclerosis. Egypt J Radiol Nucl Med 45(4):1193-1199

12. Moraal B et al (2008) Multi-contrast, isotropic, single-slab 3D MR imaging in multiple sclerosis. Eur Radiol 18(10):2311-2320

13. Bergamaschi R (2007) Prognostic factors in multiple sclerosis. Int Rev Neurobiol 79:423-447

14. Calabrese M et al (2007) Detection of cortical inflammatory lesions by double inversion recovery magnetic resonance imaging in patients with multiple sclerosis. Arch Neurol 64(10):1416-1422

15. Tremlett H, Paty D, Devonshire V (2006) Disability progression in multiple sclerosis is slower than previously reported. Neurology 66(2):172-177

\section{Publisher's Note}

Springer Nature remains neutral with regard to jurisdictional claims in published maps and institutional affiliations. 